Please use this PDF proof to check the layout of your article. If you would like any changes to be made to the layout, you can leave instructions in the online proofing interface. Making your changes directly in the online proofing interface is the quickest, easiest way to correct and submit your proof. Please note that changes made to the article in the online proofing interface will be added to the article before publication, but are not reflected in this PDF proof. 


\title{
Just-in-time learning based probabilistic gradient boosting tree for valve failure prognostics
}

\author{
Xiaochuan $\mathrm{Li}^{\mathrm{a}, *}$, David Mba ${ }^{\mathrm{a}}$, Tianran Lin ${ }^{\mathrm{b}}$, Yingjie Yang ${ }^{\mathrm{a}}$, Panagiotis Loukopoulos ${ }^{\mathrm{c}}$ \\ ${ }^{a}$ Faculty of Computing, Engineering and Media, De Montfort University, Leicester, UK \\ ${ }^{\mathrm{b}}$ School of Mechanical and Automotive Engineering, Qingdao University of Technology, Qingdao, China \\ ${ }^{\mathrm{c}}$ School of Aerospace, Cranfield University, Cranfield, UK
}

\section{A R T I C L E I N F O}

\section{Article history:}

Received 4 March 2020

Received in revised form 24 August 2020

Accepted 28 August 2020

Available online $\mathrm{xxxx}$

\section{Keywords:}

Condition monitoring

Reciprocating compressors

Prognostics

Just-in-time learning

Gradient boosting tree

\begin{abstract}
A B S T R A C T
Historical failure instances of a system with diversified degradation patterns will pose great challenge for prognostics. Consequently, it is challenging to accurately predict the remaining useful life (RUL) using a prognostic model trained from such data. To solve this problem, this paper proposes a just-in-time learning-based data-driven prognostic method for reciprocating compressors with diverse degradation patterns and operating modes. The proposed framework employs a just-in-time learning (JITL) scheme to deal with the stochastic nature of fault evolution and the diversity of degradation patterns. Moreover, a data-driven forecasting model that features a randomized and smoothed gradient boosting decision tree (RS-GBDT) is developed for RUL and uncertainty predictions. The effectiveness of the proposed approach was validated on temperature measurements collected from 13 valve failure cases of an industrial reciprocating compressor.
\end{abstract}

(c) 2020 Elsevier Ltd. All rights reserved.

\section{Introduction}

Health prognostics is at the heart of any condition-based monitoring (CBM) task, which attempts to predict the remaining useful life (RUL) of a system based on the historical and in-process degradation data. The identification and prognostics of faults has received considerable attention from both researchers in academia and engineers in industry. A number of review papers have been written on the subject [1-3].

Reciprocating compressors play a crucial role in a number of industrial applications, such as gas refineries, refrigerators and compressed natural gas stations; to name a few. These compressors account for a large portion of energy consumption in machinery sectors, thus it is crucial to ensure high levels of availability and reliability in order to reduce production cost and improve operational efficiency. Despite their popularity, owing to the fact that reciprocating compressors constitute high number of moving parts, the maintenance cost of these machines can be several times higher than that of other compressor types, and they are likely to have more failures [4]. Reciprocating compressor failures are frequently caused by breakdown of valves. It has been reported that valves account for more than one third of unplanned shutdown cases and nearly half of the total maintenance cost, rendering them one of the weakest components in a compressor [5]. Valves typically work under adverse conditions, such as rapid opening and closing, high and low flow difference, high temperatures and corrosive gases and liquids, and are thus subject to performance deterioration and failure. Therefore, it is crucial to implement effective maintenance strategies that provide accident prevention and maintenance decision-making. Despite

\footnotetext{
* Corresponding author at: Q.3.05b Queens Building, De Montfort University, UK.

E-mail address: xiaochuan.li@dmu.ac.uk (X. Li).
} 
the fact that much research has been dedicated to fault detection of reciprocating compressors, their prognostics still receive little attention.

Owing to the advances in sensing and data storage technology, massive filed data collection from machinery becomes feasible. The collected life-cycle condition data has enabled the implementation of diagnostics and prognostics using datadriven algorithms. However, historical life-cycle instances of machinery often have both diversified degradation patterns and lifespans due to a number of reasons such as various operational modes, engineering variance and environmental conditions, etc. These failure instances, if mixed together, will pose great challenges to RUL prediction. This has encouraged the CBM community to look for advanced analytical techniques. One solution is to employ the just-in-time learning (JITL) methods and develop models that are based on finding historical instances demonstrating similar degradation patterns to the testing data. Attempts have been made [6,7] to build a fault detection model using the JITL scheme which evaluates the similarity between the current data and the historical database. However, the engineering applications of these JITL-based models mainly focus on fault detection. On the other hand, instance-similarity based methods, which can be treated as another form of JITL scheme, have attracted considerable attention from researchers in the past decade [8-10]. In these methods, the RUL is estimated as a weighted-sum of the founded similar historical instances, and therefore there is a lack of model generalization and uncertainty quantification. Another solution is to employ the ensemble modelling techniques, such as boosting, to enhance the predictive performance. One approach along these lines which involves the ensemble of several predictors was proposed and tested in [11]. A GBDT-based prognostic model was proposed based on probabilistic mathematical models in [12]. Despite the fact that these methods are capable to approach the actual RUL with a high accuracy, they do not take the diversity of degradation patterns and lifespans into consideration.

In this paper, a novel JITL-data-driven prognostic model consisting of an improved JITL scheme and a randomized and smoothed gradient boosting decision tree (RS-GBDT) is proposed. The JITL scheme accounts for the diversified degradation patterns and various operational modes and facilitates the subsequent predictive model by grouping the instances with similar degradation patterns together. The RS-GBDT approach employs a randomized and smoothed gradient and inherits the merits of high predictive accuracy and narrow probabilistic output. temperature measurements obtained from an inservice reciprocating compressor are utilized to verify the effectiveness of the proposed model.

The main contributions of this work are summarized as follows:1) proposes a JITL-data-driven prognostic model for inservice reciprocating compressors with diversified degradation patterns and various operational modes; 2) develops a JITL scheme which realizes time-series clustering without knowing the number of cluster centers; 3 ) develops a RS-GBDT model with improved predictive performance and uncertainty quantification; 4) Presents the degradation data of a large-scale inservice compressor.

A brief outline of remaining parts of the paper is as follows: In Section 2, related algorithms and the proposed prognostic model are presented in details. In Section 3 the experimental data is explained, and experimental results as well as detailed discussions about the results are presented. Conclusion remarks are provided in Section 4.

\section{Methodology}

The proposed JITL-RS-GBDT prognostic scheme has been realized in two stages. The first stage is based on an improved JITL scheme, which takes the failure trajectories (both historical and up-to-date query data) as inputs and returns the similar failure cases as output. The second stage consists of an improved version of GBDT, which predicts the RUL of the query machine based on the similar trajectories found in the first stage. A brief description of the JITL method is provided in Subsection A. The proposed JITL scheme is detailed in Subsection B. The RS-GBDT is presented in Subsection C. Subsection D presents a synthesis of the proposed prognostic scheme.

\subsection{Conventional just-in-time learning scheme}

Owing to the stochastic nature of fault evolution and the mixture of different operation modes, historical failure data with various degradation patterns will be mixed. In this paper, a JITL scheme based on time-series clustering is proposed to account for this various degradation patterns. Unlike other online monitoring methods which require model updating from scratch for online adaptation, JITL is inherently adaptive in nature. JITL builds a local model by the most similar data to the query data in contrast to the traditional global modeling methods, which relies on the complete training dataset.

With a current query data $z_{q}$, there are three main steps in a JITL scheme to build a model on-line:

1) Retrieve the relevant historical data $z_{i}$ that are similar to the query data $z_{q}$ from the training database according to the nearest neighborhood criterion.

2) Build a local model using the retrieved data.

3) Obtain the output based on the local model, then discard the local model and build a new local model in the same way when a new query data becomes available. 


\subsubsection{Related work}

Retrieving the relevant data typically involves two major steps. The first step is to specify a pairwise measure of similarity between series. Nearest neighborhood criterion which is based on the similarity between the historical data $z_{i}$ and the query data $z_{q}$ is often employed for this task. Euclidean norm $d\left(z_{q}, z_{i}\right)=\left\|z_{q}-z_{i}\right\|_{2}$ is frequently used as a distance measure [13]. Moreover, some researchers developed methods that involve solving an optimization problem to find a best match between the historical data $z_{i}$ and the query data $z_{q}$, such as dynamic time warping (DTW) [14]. Although DTW has been found to be useful for time series matching in the field of speech processing, it may not be applicable to RUL prediction since it considers data with similar degradation patterns but different lifespans to be similar. In RUL prediction, however, relevant trajectories are assumed to have both similar degradation patterns and lifespans.

The second step to retrieve the relevant data is to pass the similarity measure to an algorithm which determines associations between the trajectories. To mention a few examples, centroid-based techniques such as k-means clustering, see [15]. K-means requires a certain number of cluster centers to be determined in advance, which could be difficult to achieve especially when all the failure cases are from the same failure mode. Hierarchical methods such as agglomerative clustering, see for example [16]. Similar to k-means, users need to manually define the number of clusters. Some researchers proposed to use the similarity measure to sort out the relevant data, for instance in [6], all relevant series $z_{i}$ are sorted in a descending order according to the numerical value of $d_{-} c\left(z_{q}, z_{i}\right)$, and the first $l$ number of $z_{i}$ are determined through an optimization procedure. This method is cumbersome since it involves the evaluation of all possible numbers of $l$ for each individual series $z_{i}$. In [17], All relevant series $z_{i}$ are utilized for the training of the prognostic model. However, failure cases that demonstrate different degradation patterns should be eliminated from the training stage, and that's the reason why a JITL scheme is presented in this paper. The above-mentioned methods either require the number of clusters to be determined beforehand or the optimal number of relevant series to be tested iteratively.

\subsection{Improved just-in-time learning scheme}

In this paper, we propose an improved JITL scheme which accounts for both distance and spatial similarities and features the ability to group failure trajectories together without knowing the number of cluster centers. The details of this scheme are described as follows.

Step 1: Pairwise measure of similarity between series

In this study, a distance measure which considers both distance similarity and spatial direction similarity is developed.

The Cosine similarity, which measures the spatial direction similarity between two series, is often neglected by the prognostics community [8]. The Cosine similarity is defined as:

$$
\cos \left(z_{q}, z_{i}\right)=\frac{\boldsymbol{z}_{\boldsymbol{q}} \cdot \boldsymbol{z}_{\boldsymbol{i}}}{\left\|\boldsymbol{z}_{\boldsymbol{q}}\right\|_{2}\left\|\boldsymbol{z}_{\boldsymbol{i}}\right\|_{2}}
$$

where $\boldsymbol{z}_{\boldsymbol{q}}$ denotes the query data and $\boldsymbol{z}_{\boldsymbol{i}}$ denotes the reference data. $\left\|\boldsymbol{z}_{\boldsymbol{q}}\right\|_{2}$ denotes the Euclidean norm of $\boldsymbol{z}_{\boldsymbol{q}}$, defined as $\sqrt{z_{q, 1}{ }^{2}+z_{q, 2}{ }^{2}+\cdots+z_{q, N}{ }^{2}}\left(N\right.$ is the number of elements in vector $\boldsymbol{z}_{\boldsymbol{q}}$ ). Similarly, $\left\|\boldsymbol{z}_{\boldsymbol{i}}\right\|_{2}$ denotes the Euclidean norm of $z_{i}$, defined as $\sqrt{z_{i, 1}^{2}+z_{i, 2}^{2}+\cdots+z_{i, N}^{2}}$.

Therefore, the pairwise similarity between series which accounts for both distance and spatial similarity is defined as follows.

$$
d_{-} c\left(\boldsymbol{z}_{\boldsymbol{q}}, \boldsymbol{z}_{\boldsymbol{i}}\right)=\left(\sqrt{e^{-d\left(\boldsymbol{z}_{\boldsymbol{q}}, \boldsymbol{z}_{\boldsymbol{i}}\right)}}+\cos \left(\boldsymbol{z}_{\boldsymbol{q}}, \boldsymbol{z}_{\boldsymbol{i}}\right)\right)
$$

Step 2: Determination of associations between the series

The second step to retrieve the relevant data is to pass the similarity measure to an algorithm to determine associations between the trajectories. In this paper, the density Based Spatial Clustering of Applications with Noise (DBSCAN) algorithm [18], which is a density-based clustering algorithm, is adopted to form the second part of the proposed JITL scheme. DBSCAN is able to accurately recognize clusters of arbitrary size, making it suitable for the online determination of query-relevant data because the cluster sizes may evolve and change during the fault evolution. Another benefit of DBSCAN is that it can find the relevant failure data without the predefined clusters' number.

Given a group of points, DBSCAN groups together points that are considered to be close to each other according to two parameters, a minimum number of neighborhoods minPints, and the radius $\epsilon$. A point is considered to be a core point if it has more than minPints number of neighborhoods within the radius $\epsilon$. The scanning process of DBSCAN starts by picking an arbitrary point $P$ from the database. If $P$ is determined as a core point, $P$ and its neighbors within the $\epsilon$-radius form a new circle. All points in the circle are scanned and their corresponding $\epsilon$-radius neighbors are included in the circle. The newly added points are also expanded to see if their $\epsilon$-radius neighbors can be included in the circle. If any of these points within the radius is found again to be a core point, their neighbors are transitively included. This process is implemented recursively until no more points can be included in the circle. During the scanning process, points that have been assigned to a cluster will not be scanned again when encountered later by the scanning process. Points which are non-core points are considered as noise. Noise points are not assigned to any clusters. Then, DBSCAN moves to the next un-scanned point and the scanning process stops when all points have been scanned. A pseudo-code for the DBSCAN algorithm is demonstrated in Algorithm 1 . 

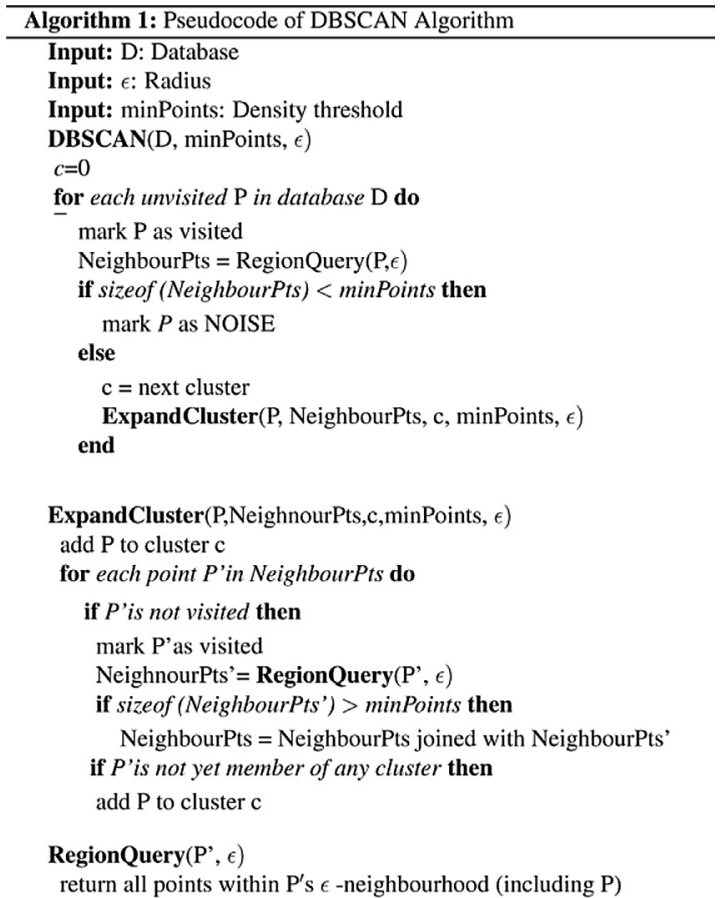

In summary, the proposed JITL scheme first calculates a distance measure which considers both distance similarity and spatial direction similarity between the testing and historical instances. The distance measure is computed between each pair of instances such that a similarity matrix of size $13^{*} 13$ is obtained (13 is the total number of failure instances). This distance matrix is updated iteratively once a new set of measurement on the testing machine become available. Meanwhile, this distance matrix is passed to the DBSCAN algorithm which determines associations between the instances. DBSCAN will start at a random point (each point represents a failure instance, e.g. there are 13 points in total), and it will count how many other points are nearby. DBSCAN will continue this process until no other data points are nearby, and then it will look to form a second cluster. DBSCAN will stop searching for new clusters until all points have been properly allocated. The proposed JITL scheme iteratively realizes clustering of time-series instances without knowing how many cluster centres are needed.

\subsection{Improved gradient boosting trees}

Data driven prognostic techniques fall into two main categories: techniques which are built based on one strong predictive model, such as [19] and techniques built based on a couple of week predictors and make an ensemble prediction [12,20]. The ensemble method usually leads to better predictive performance compared to that being achieved by any individual weak predictor. Bagging [21] and Boosting [22] are two powerful ensemble methods that are commonly utilized to combine weak learners. Bagging, also known as Bootstrap aggregating, is realized in two stages. At the first stage, a number of bootstrapped subsamples are pulled and a week learner is constructed based on each of the subsamples. At the second stage, the aggregation averages over the weak learners to achieve the most efficient predictor. Perturbing the training data can significantly improve the predictive accuracy as it brings substantial changes to the predictor. Bagging has been applied to various areas, and the Random forests has been one of the most prominent examples [23]. Boosting, on the contrary, aims to generate a strong learner from a set of weak learners. In boosting methods, a weak learner is iteratively added to the ensemble, which is trained against the error of the whole ensemble. The first implementation of boosting that witnessed great success in application was AdaBoost [24]. The base learners used in AdaBoost are decision trees with a single split. AdaBoost was later on recast in a statistical framework [25] and was further improved by Friedman and called Gradient Boosting Machines/Trees [26]. Gradient boosting trees (GBDT) has gained popularity and momentum in data-science competitions such as Kaggle.

Given a training sample $D=\left\{\left(z_{i}, y_{i}\right)\right\}_{i=1}^{N}$, a GBDT attempts to predict the output $y_{i}$ for a given input $z_{i}$ based on the mappings from input to output that are learnt during the training stage. For the case of regression problems, the base learners are regression trees. Regression trees are built through a process known as binary recursive partitioning, which is a recursive process that partitions the data space into disjoint branches, and then continues partitioning each branch into smaller regions. Regression trees can be formulated as follows.

$$
R T_{N}\left(\boldsymbol{z}, \theta_{j}\right)=\sum_{i=1}^{N} \frac{y_{i} I_{z_{i} \in R_{N}\left(\boldsymbol{z}, \theta_{j}\right)}}{\sum_{i=1}^{N} I_{z_{i} \in R_{N}}\left(\boldsymbol{z}, \theta_{j}\right)}
$$


where $R_{N}\left(\boldsymbol{z}, \theta_{j}\right)$ refers to the individual disjoint branch, $\theta_{j}$ represents the tree model parameters and $I$ is the indicator function. A GBDT uses M additive regression trees to predict the output:

$$
f_{Z}\left(\boldsymbol{z}, \theta_{j=1}^{M}\right)=\frac{1}{Z} \sum_{j=1}^{M} R T_{N}\left(\boldsymbol{z}, \theta_{j}\right)
$$

where $M$ represents the total number of regression trees and $R T_{N}\left(\boldsymbol{z}, \theta_{j}\right)$ represents the space of the $j$ th regression tree, and $z$ is the input data. The loss function for evaluating the split candidates is given by

$$
\mathcal{L}_{\text {split }}=\frac{1}{2}\left(\frac{\left(\sum_{i \in I_{L}} g_{i}\right)^{2}}{\sum_{i \in I_{L}} h_{i}+\lambda}+\frac{\left(\sum_{i \in I_{R}} g_{i}\right)^{2}}{\sum_{i \in I_{R}} h_{i}+\lambda}-\frac{\left(\sum_{i \in I} g_{i}\right)^{2}}{\sum_{i \in I} h_{i}+\lambda}\right)-\gamma
$$

where $I_{L}$ and $I_{R}$ are the instance sets of left and right nodes after the split. $g_{i}$ and $h_{i}$ represent the gradient and second order gradient/hessian statistics on the loss function $\mathcal{L}_{\text {split. }} \lambda$ represents the $\mathrm{L} 2$ regularization term, $\gamma$ is the minimum loss reduction required to make a split. A GBDT recursively searches the potential split candidates according to (5) and adds the best split to the tree until the maximum tree depth is reached.

Since prognostics involves predicting at future times the RUL using a dynamic model without true measurements, it may inevitably result in large gain of uncertainty. For the purpose of characterizing the precision of the prediction, this uncertainty needs to be estimated together with the RUL. A way to quantify this prediction uncertainty in the context of a GBDT is through quantile regression which attempts to assess the conditional quantiles from the observed data [27]. The conditional quantiles can be calculated through minimizing the following loss function

$$
C\left(z_{i}-q\right)=\min _{q}\left\{\begin{array}{lll}
(\alpha-1)\left|z_{i}-q\right| & \text { if } & z_{i}<q \\
\alpha\left|z_{i}-q\right| & \text { if } & z_{i}>q
\end{array}\right.
$$

where $z_{i}$ is the observation, $q$ denotes the quantile estimate, and $\alpha, 0<\alpha<1$ is the quantile range. To visualize (6), Fig. 1 (a) and (b) illustrates the loss function of quantile regression for $\alpha=0.95$. In the figure, the $\mathrm{x}$-axis represents the deviation

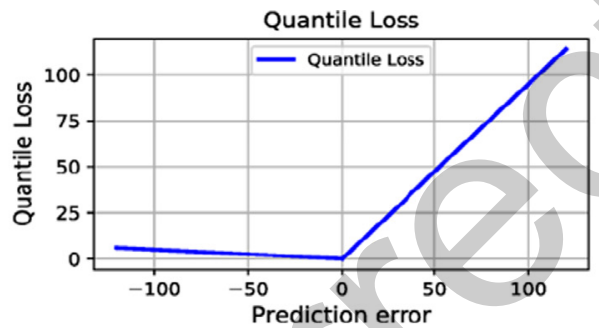

(a)

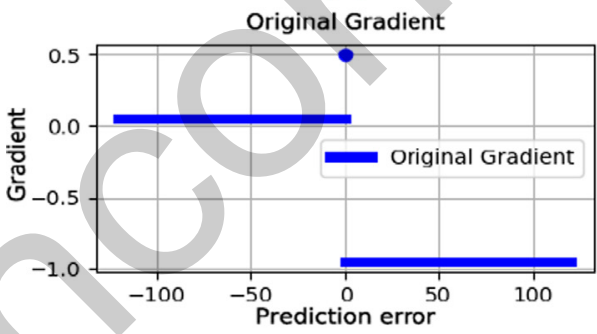

(c)

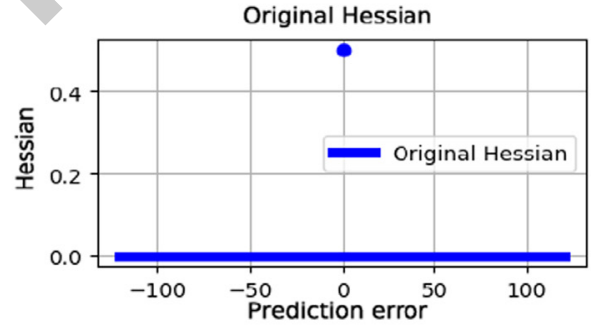

(e)

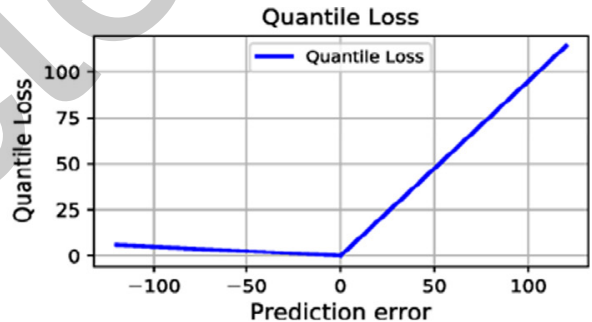

(b)

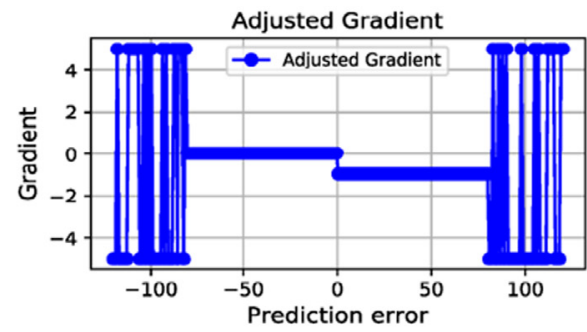

(d)

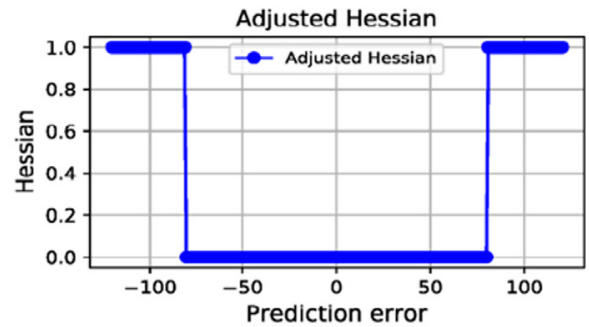

(f)

Fig. 1. Comparison between the original and modified gradient and the second order gradient on the quantile loss function. 
$\left|z_{i}-q\right|$ between the observation and the quantile estimate. The resultant gradient $g_{i}$ and second order gradient statistics $h_{i}$ are plotted in Fig. 1 (c) and (e), respectively. $g_{i}$ can be formulated as

$$
g_{i}= \begin{cases}1-\alpha & \text { if } \quad x<0 \\ 0.5 & \text { if } x=0 \\ -\alpha & \text { if } x>0\end{cases}
$$

As a result, the second order gradient $h_{i}$ can be formulated as

$$
h_{i}=\left\{\begin{array}{lll}
0 & \text { if } & x \neq 0 \\
0.5 & \text { if } & x=0
\end{array}\right.
$$

where $x=\left|z_{i}-q\right|$.

In the case that the deviation $\left|z_{i}-q\right|$ is relatively large within the partition, then since the gradient $g_{i}$ and the second order gradient $h_{i}$ both being constant for large values of $\left|z_{i}-q\right|$, the splitting score stays zero and no split occurs. In order to solve this problem, $g_{i}$ and $h_{i}$ are modified to lead to better partitioning and therefore better predictive performance. The modified gradient $g_{i}$ is given by

$$
g_{i}^{*}=\left\{\begin{array}{ccc}
q_{\beta} & \text { if } x<-q_{\varepsilon} \\
1-\alpha & \text { if } & -q_{\varepsilon}<x<(\alpha-1) q_{\delta} \\
-\frac{x}{q_{\delta}} & \text { if } & (\alpha-1) q_{\delta}<x<\alpha q_{\delta} \\
-\alpha & \text { if } \alpha q_{\delta}<x<q_{\varepsilon} \\
q_{\beta} & \text { if } q_{\varepsilon}<x
\end{array}\right.
$$

where $q_{\beta}, q_{\varepsilon}$ and $q_{\delta}$ are hyperparameters that control the randomization and smoothness of the modified gradient. $q_{\beta}$ randomly takes either one of the two values $\left|q_{\beta}\right|$ or $-\left|q_{\beta}\right|$ and this randomization can force a random split when the deviation $x$ is relatively large. Moreover, $-\frac{x}{q_{\delta}}$ is used to smooth the original gradient when $x$ is close to the origin such that the gradient is not always constant. Compared with the original gradient, such a modification can increase the number of tree splits when the difference between the observation and the quantile volume is relatively large and close to zero. Consequently, we expect to see a more "sensitive" GBDT which can result in better prognostics as well as narrowed confidence boundaries. The modified gradient has also led to a modified second order gradient $h_{i}^{*}$, which is formulated as

$$
h_{i}^{*}=\left\{\begin{array}{ccc}
1 & \text { if } x<-q_{\varepsilon} \\
0 & \text { if } & -q_{\varepsilon}<x<(\alpha-1) q_{\delta} \\
-\frac{1}{q_{\delta}} & \text { if } & (\alpha-1) q_{\delta}<x<\alpha q_{\delta} \\
0 & \text { if } \alpha q_{\delta}<x<q_{\varepsilon} \\
1 & \text { if } q_{\varepsilon}<x
\end{array}\right.
$$

$g_{i}^{*}$ and $h_{i}^{*}$ are illustrated in Fig. 1 (d) and (f), respectively.

The $95 \%$ and $5 \%$ confidence boundaries are calculated by setting the parameter $\alpha$ in (9)-(10) to 0.95 and 0.05 , respectively.

\subsection{Synthesis of the proposed prognostic method and RUL prediction}

Based on the algorithms addressed in Sections A-C, a data-driven prognostic framework called JITL-RS-GBDT is proposed in this study.

The JITL-RS-GBDT approach combines a JITL scheme and a data-driven prognostic method. Firstly, the JITL scheme as proposed in Section B serves as time-series clustering and accounts for various operational modes and the stochastic nature of fault evolution. Given a query data, the JITL scheme finds the relevant data which share the similar degradation patterns. Then the data-driven prognostic method as proposed in Section C is utilized to predict RUL based on the relevant data found through the JITL scheme.

The pairwise similarities between the current sample data and the historical failure data are firstly calculated with (2). Then the calculated similarity matrix serves as the input of the DBSCAN, which then finds the relevant failure cases that exhibit similar degradation patterns. These relevant failure data along with the corresponding RULs then serve as the input of the RS-GBDT method for RUL prediction. The above-mentioned procedure is repeated recursively once a new sample data becomes available, thereby owning inherent online adaptation. The original data are preprocessed as per the processes detailed in [12] where the Hoteling $T^{2}$ index was used to represent the fault evolution. The implementation procedure of the JITL-RS-GBDT approach is illustrated in Fig. 2. Fig. 3 also lists the advantages of the proposed prognostic scheme. 


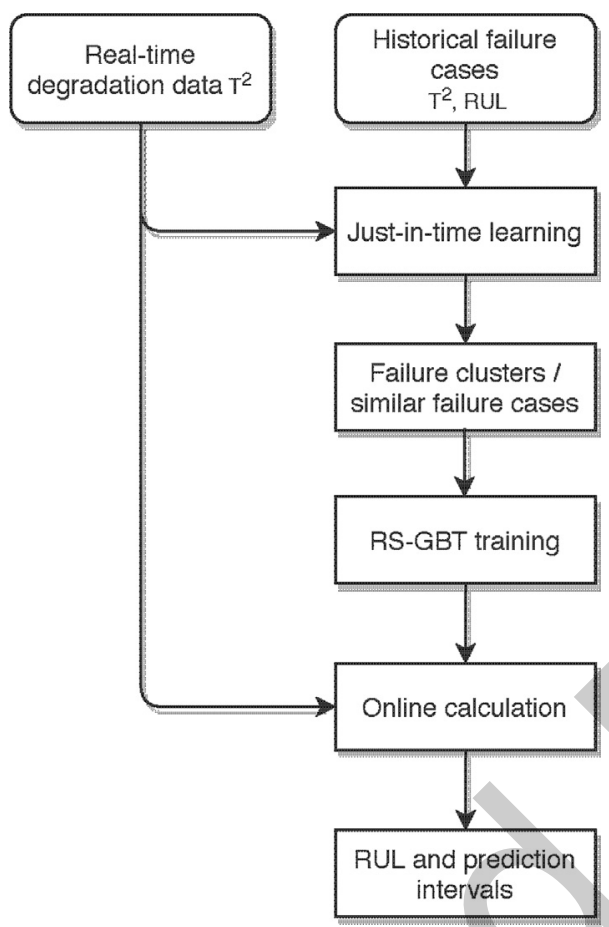

Fig. 2. Implementation of the JITL-RS-GBDT prognostic scheme.

\section{Improved JITL scheme}

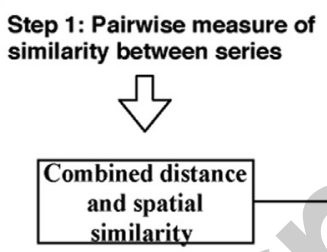

\section{RS-GBDT}

Step 2: Determination of associations between the series

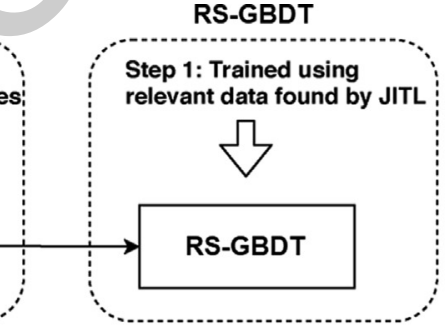

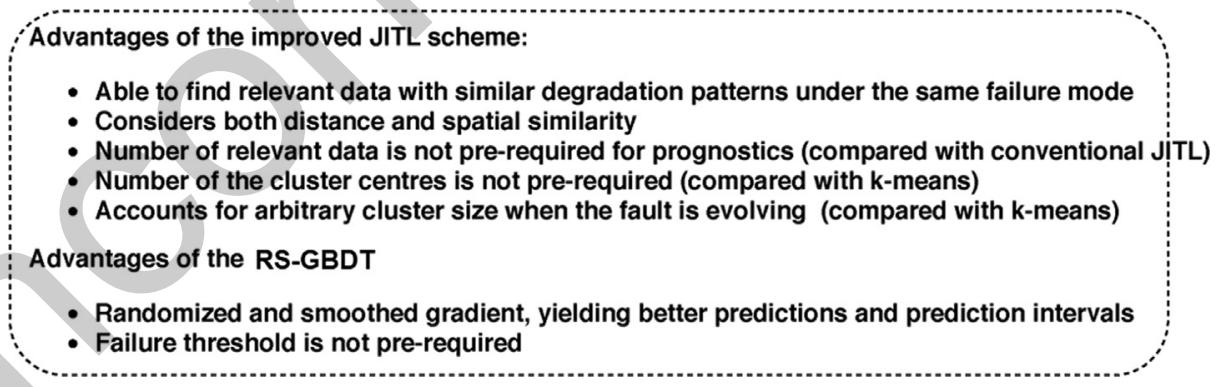

Fig. 3. Key features of the proposed JITL-RS-GBDT prognostic framework.

\subsection{Description of dataset}

In this section, data captured from a two-stage, four-cylinder, double-acting operational reciprocating compressor are presented. The machine experienced thirteen valve failures within a period of 18 months with all failures took place at the fourth cylinder. The root cause of these failures was found to be improper sealing of the valve due to a missing piece from the outer structure of valve plate. These failures occurred at either the head end (HE) or the crank end (CE) discharge 
valve. Only temperature measurements were recorded by the monitoring system. Eight temperature ratios, namely Suction temperature $\mathrm{HE} / \mathrm{CE}$ cylinder 1-4 and Discharge temperature HE/CE cylinder 1-4 were utilized by the site engineer for monitoring the health status of the valves.

Similar to [12], Principal component analysis (PCA) was employed to merge the data from the sensors, resulting in the Hoteling $T^{2}$ index which represents the health status of the machine. In order to train the PCA model, a substantial amount of normal data was collected from each failure case. Around 20,17 and $24 \mathrm{~h}$ of healthy data were obtained from cases 2,4 and 12 , respectively. Around $36.1 \mathrm{~h}$ of healthy data were collected for failure cases 1,3,5-11 and 13 . These data were utilized during the training stage and the trained PCA was employed to create the $T^{2}$ indices. Fig. 4 illustrates the index trend for all failure cases. The number of the principal components selected was such that at least a percentage of $95 \%$ of the total variance is explained by them, thus reducing the number of variables. In all cases the first three components were sufficient. The significance level for computing $T^{2}$ was set to 0.01 .

Failure times demonstrate individual variations between failure cases highlighting the stochastic nature of fault evolution in real-world applications. Although all thirteen cases were captured under the same failure condition, these trajectories demonstrate various degradation patterns, with the shortest lifespan being 73 and the longest being 494 . these variations were mainly caused by the different applications the compressor has experienced (the machine compressed various types of gases in the past). Failure times vary in duration and limited amount of failure cases (13 failure cases in total) have made forecasting the FT of this machine a complicated challenge. We thereby use this dataset to test the superiority of the proposed method.

\subsection{RUL prediction and analysis}

In the online prognostic phase, the $T^{2}$ index of the current sample data (query data) were fed together with those of the historical data into the JITL scheme. The JITL method will perform clustering based on the spatial and distance similarities between the failure cases and find the data that are most relevant to the query data in terms of the fault degradation patterns. The next stage of the prognostic scheme involves feeding the $T^{2}$ index together with the true RUL of the relevant cases into the RS-GBDT model in order to train the model. Then the trained predictor is employed to predict the RUL of the query data based on the up-to-date $T^{2}$ index values.

The hyperparameters of the JITL-RS-GBDT scheme were tuned as follows. The DBSCAN model was trained with the minPints set to 2. In other words, the minimum number of instances in any cluster was set to two. This is to ensure there exists at least one historical instance in the cluster where the query data lies in, such that the GBDT predictor can be trained on no less than one similar historical instance. The optimal value of the radius $\epsilon$ was tuned offline based on the historical failure data. $\epsilon$ was chosen to ensure that at each iteration the maximum number of "noise cases" was no greater than one. The hyperparameters for both the original GBDT and the RS-GBDT models were tuned using Random Grid Search with 5 -fold cross-validation. As a result, the maximum tree depth for base learners was 5 and the optimal learning rate was 0.03 ,

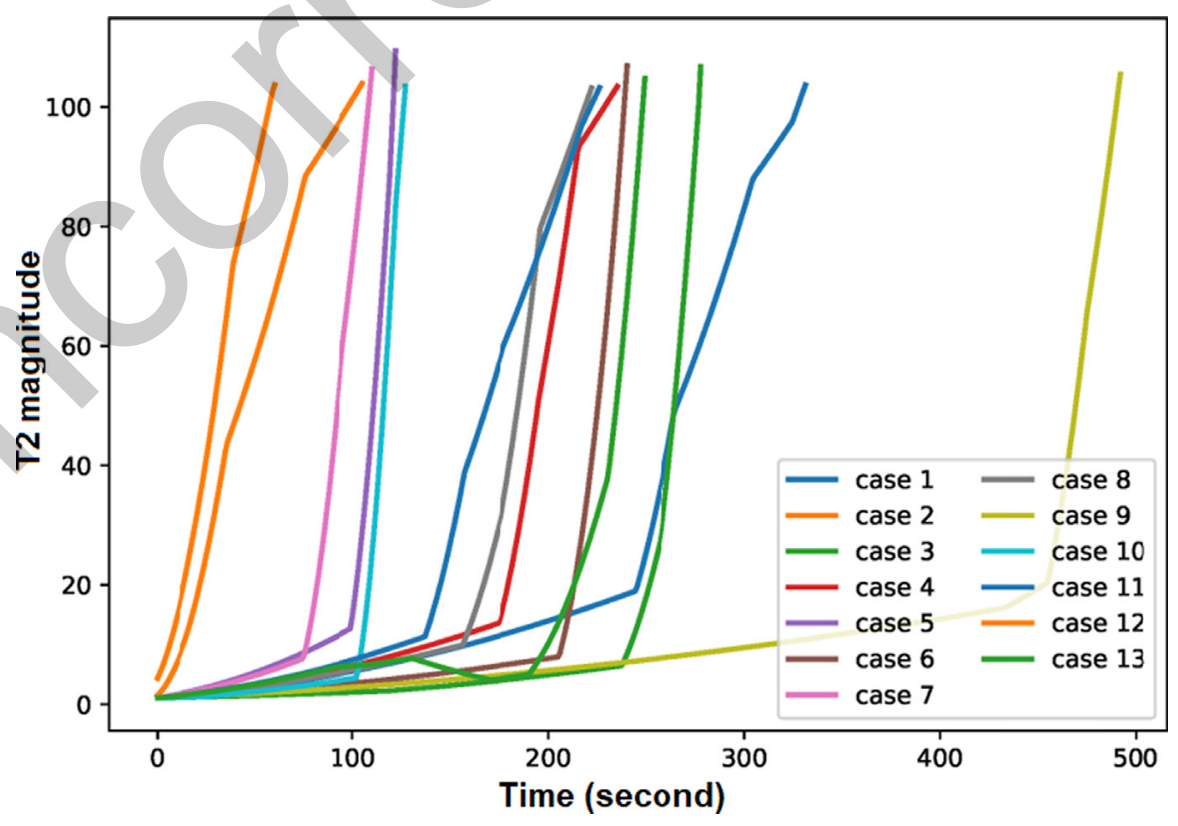

Fig. 4. Hoteling $T^{2}$ index trend for all failure cases. 
and the number of base learners was set to 200. For a fair comparison, the abovementioned hyperparameters were set to be the same for both GBDT and the RS-GBDT. The three additional hyperparameters $q_{\delta}, q_{\varepsilon}$ and $q_{\beta}$ that control the randomization and smoothness of the RS-GBDT model were selected also using Random Grid Search.

The proposed prognostic algorithm is capable of providing an estimation in less than a specific time window set in this work at $4 \mathrm{~s}$. Compared with the long prediction timeframes (machine total RULs are ranging from 61 to $493 \mathrm{~s}$ ), the processing time of the proposed method is deemed to be relatively short, which confirms its applicability for online monitoring. In the online phase the developed algorithms provided with predictions within the specified time window with calculations taking place on a desktop computer with a $3.2 \mathrm{GHz} 8$ core CPU and $16 \mathrm{~GB}$ memory.

The prognostic results of JITL-RS-GBDT scheme in cases 1-13 are illustrated in Fig. 5. The results obtained by JITL-GBDT and GBDT are also presented for comparison. The GBDT model apparently resulted in overestimated RUL for cases 3, 5, 6, 7 and 10. It also generated underestimated RUL predictions for case 2 . This is mainly due to a rapid degradation taking place within short time which makes the underlying dynamics difficult to be captured by the GBDT model. These observations are coincide with the results reported in [12]. The JITL models, however, overcome the aforementioned problem, therefore properly captured the degradation patterns in these situations where abrupt rises took place. For cases 1, 4, 8, 9, 11, 12 and 13 , quantitative analysis was carried out to assess the prognostic performance of the three models (see Table 1). It can be observed from Table 1 that the proposed JITL scheme is able to significantly increase the predictive accuracy, in most failure cases. This is because the cases that have similar lifespan tend to have similar degradation patterns during fault evolution (see Fig. 4), meaning that those cases being grouped together by the JITL scheme are likely to have similar failure time, which can facilitate the subsequent data-driven prognostic model. The diversity of degradation patterns has resulted in various fault evolution trajectories with diversified lifespans. In our previous publication [1], all historical failure instances with var-

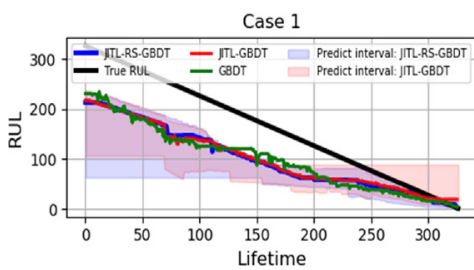

Case 4
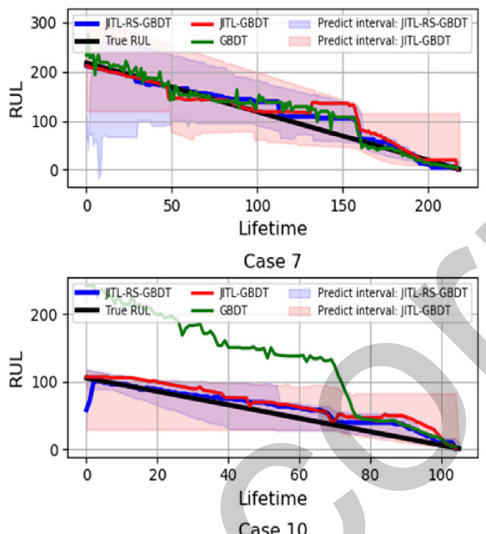

Case 10

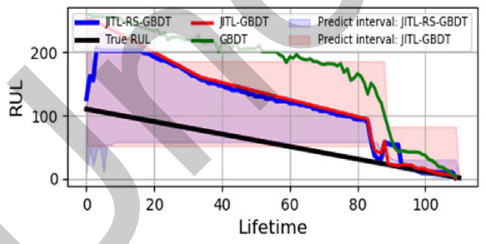

Case 13

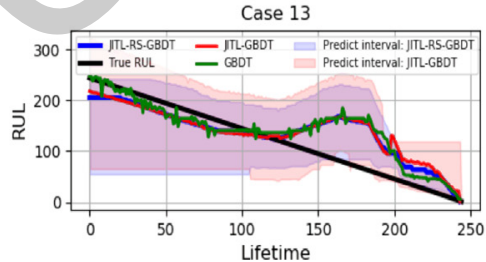

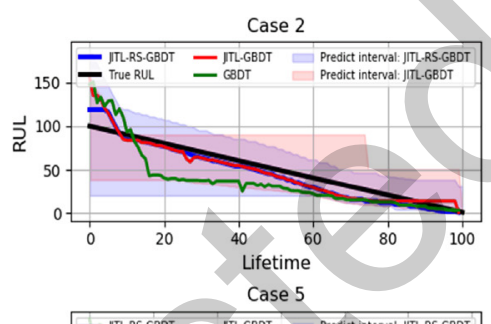
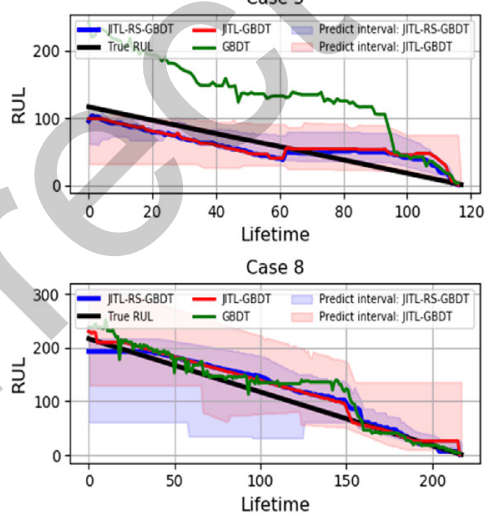

Case 11

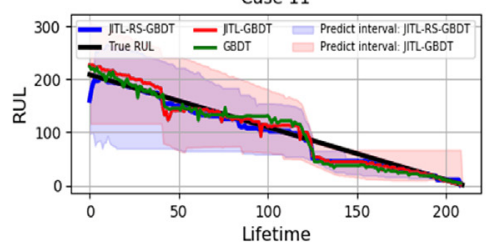

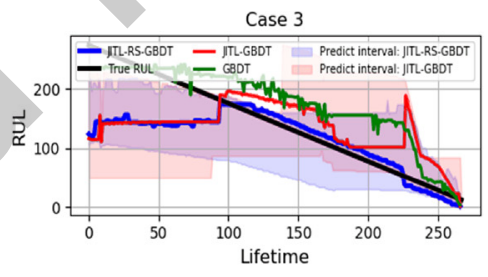

Case 6
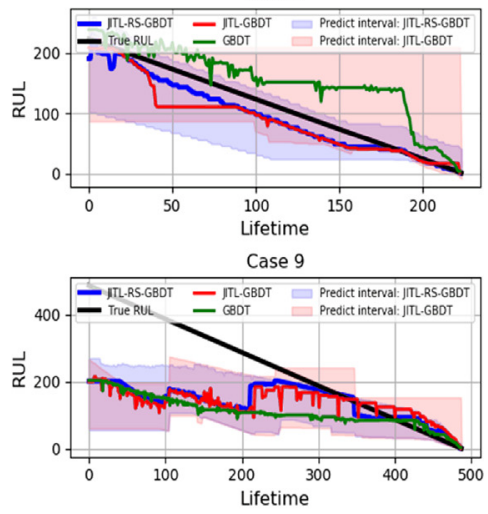

Case 12

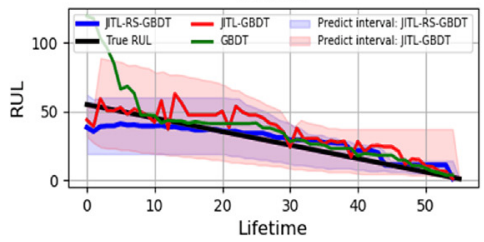

Fig. 5. Predictive performance for fault cases $1-13$. The shaded areas denote the $95 \%$ prediction intervals. 
Table 1

Comparison of predictive accuracy.

\begin{tabular}{|c|c|c|c|c|c|c|c|c|c|c|}
\hline \multirow{2}{*}{$\begin{array}{l}\text { Failure } \\
\text { Cases }\end{array}$} & \multicolumn{5}{|l|}{ MAE } & \multicolumn{5}{|l|}{ RMSE } \\
\hline & A & в & c & d & $\mathrm{e}$ & $\mathrm{a}$ & $\mathrm{b}$ & c & $\mathrm{d}$ & $\mathrm{e}$ \\
\hline Case 1 & 63.7 & 63.4 & 63.3 & 63.33 & 62.73 & 71.8 & 72 & 71 & 70.92 & 68.9 \\
\hline Case 2 & 7.6 & 8.7 & 19.9 & 19.74 & 20.4 & 8.9 & 11.7 & 23.2 & 23.25 & 24.16 \\
\hline Case 3 & 40.7 & 59.3 & 47.3 & 47.19 & 56.17 & 57 & 67.1 & 54.5 & 54.5 & 64.08 \\
\hline Case 4 & 11.9 & 15.5 & 17.3 & 15 & 59.39 & 16.1 & 24 & 29.1 & 20.48 & 69.59 \\
\hline Case 5 & 15.9 & 17.8 & 75.1 & 74.52 & 80.05 & 17 & 19.3 & 79.8 & 79.76 & 80.32 \\
\hline Case 6 & 22.6 & 23.8 & 41.9 & 41.75 & 47.01 & 28.2 & 42.4 & 49 & 49.03 & 53.53 \\
\hline Case 7 & 9.9 & 15.8 & 78.2 & 77.45 & 82.06 & 12.2 & 17.2 & 88.1 & 88.12 & 92.76 \\
\hline Case 8 & 15.8 & 14.7 & 19.5 & 19.41 & 31.3 & 18.6 & 17 & 25.6 & 25.6 & 35.64 \\
\hline Case 9 & 110 & 120.6 & 137.6 & 137.3 & 132.8 & 149.7 & 157.2 & 166.6 & 166.6 & 162.4 \\
\hline Case 10 & 65.3 & 79.7 & 122.3 & 121.3 & 125.6 & 73.7 & 90.9 & 129.8 & 129.8 & 132.4 \\
\hline Case 11 & 8.1 & 11.5 & 12.7 & 12.91 & 15.7 & 11.7 & 14.7 & 16 & 16.17 & 18.59 \\
\hline Case 12 & 5.9 & 9.2 & 10 & 10.11 & 12.91 & 7 & 10.5 & 17.2 & 29.35 & 19.65 \\
\hline Case 13 & 33.8 & 36.1 & 31.1 & 30.96 & 31.53 & 42.1 & 44.1 & 42.6 & 40.52 & 44.34 \\
\hline Failure & MAPE & & & & & SCORE & & & & \\
\hline Cases & A & B & c & d & e & $\mathbf{a}$ & b & c & d & e \\
\hline Case 1 & 0.395 & 0.407 & 0.403 & 0.408 & 0.527 & 11.03 & 11.56 & 14.98 & 15.47 & 14.67 \\
\hline Case 2 & 0.222 & 0.345 & 0.402 & 0.411 & 0.67 & 1.22 & 1.12 & 1.26 & 1.69 & 1.56 \\
\hline Case 3 & 0.256 & 0.821 & 0.678 & 0.698 & 1.01 & 2.47 & 1.10 & 14.26 & 16.18 & 33.71 \\
\hline Case 4 & 0.179 & 0.34 & 0.402 & 0.193 & 2.76 & 1.64 & 1.99 & 3.11 & 2.21 & 52.42 \\
\hline Case 5 & 0.531 & 0.71 & 1.46 & 1.6 & 3.69 & 1.1 & 1.02 & 167 & 143.7 & 207.7 \\
\hline Case 6 & 0.19 & 0.29 & 0.712 & 0.717 & 0.889 & 2.76 & 2.46 & 5.8 & 15.97 & 22.97 \\
\hline Case 7 & 0.409 & 0.639 & 1.39 & 1.5 & 2.03 & 1.71 & 2.85 & 31.06 & 33.09 & 237.6 \\
\hline Case 8 & 0.19 & 0.346 & 0.236 & 0.24 & 1.18 & 2.324 & 2.61 & 2.126 & 3.20 & 8.06 \\
\hline Case 9 & 0.414 & 0.532 & 0.514 & 0.523 & 0.573 & 84.52 & 91.12 & 323.6 & 320.4 & 247.5 \\
\hline Case 10 & 1.13 & 1.22 & 2.19 & 2.34 & 3.27 & 36.09 & 194.9 & 3089 & 3250 & 4318 \\
\hline Case 11 & 0.178 & 0.196 & 0.211 & 0.183 & 0.502 & 1.87 & 1.91 & 2.02 & 1.11 & 1.33 \\
\hline Case 12 & 0.402 & 0.517 & 0.55 & 0.433 & 1.15 & 1.05 & 1.66 & 2.23 & 1.92 & 2.36 \\
\hline Case 13 & 0.60 & 0.74 & 0.57 & 0.544 & 0.793 & 2.83 & 3.46 & 2.69 & 2.65 & 5.47 \\
\hline
\end{tabular}

* Best results are highlighted in bold.

*a: JITL-RS-GBDT; b: JITL-GBDT; c: GBDT; d: XGBoost; e: LSTM.

ious degradation patterns are used to train a predictor, thereby resulted in unsatisfied prediction results. In this paper, the improved JITL scheme selects instances with similar degradation patterns and lifespans based on the similarity index calculated from up-to-date failure data. Historical instances that are considered to be close to each other are grouped together and utilized to train a predictor. In this way, the proposed JITL-predictor scheme has achieved an improved predictive accuracy compared with traditional GBDT.

In Table 1, four different metrics mean absolute error (MAE), root mean square error (RMSE), mean absolute percentage error (MAPE) and SCORE [28] were adopted to evaluate the predictive performance of the various prognostic models, where a, b, c, d, e stand for JITL-RS-GBDT, JITL-GBDT, GBDT, XGBoost [29] and LSTM [30]. The proposed method achieves the best performance in terms of MAE, RMSE and MAPE in most failure cases. Moreover, the averaged SCORE for the five prognostic methods considered in this study were 11.59, 24.44, 281.5, 292.9 and 398.1, respectively.

Both JITL-RS-GBDT and JITL-GBDT models are able to approach the actual RUL in most failure cases. Surprisingly, case 12 , which is the left outlier failure case with the shortest lifespan, was well captured by the JITL-RS-GBDT model. It is observed that the experimental performances of case 1 and case 9 are not ideal. This is due to the fact that cases 1 and 9 are the longest trajectories (i.e. the right outliers) among all failure cases (see Fig. 4), and hence the similar instances found by JITL are likely to be much shorter than those of cases 1 and 9 . As a consequence, case 9 is initially underestimated but captured well at the last half of its lifetime, making the predictions more accurate than the results obtained using the traditional GBDT as shown in [12]. Case 1, however, is constantly underestimated until the last quarter of its lifetime. In order to avoid domination of the results with higher absolute values generated by GBDT in Fig. 5, the comparison between JITL-RS-GBDT and JITL-GBDT are mainly carried out through quantitative analysis. Table 1 confirms that collectively JITL-RS-GBDT resulted in better predictive performance than JITL-GBDT, which can be attributed to the modified gradient and second order gradient.

Based on the information provided by Fig. 5, it can be observed that, for cases 1, 3, 5, 6 and 10, the predicted RUL occasionally decreases with lifetime linearly. This is mainly due to the following reasons. 1) the nature of failure evolution. It is observable from Fig. 4 that most of the historical failure instances involve a two-stage damage evolution, namely a slow evolution stage before the $\mathrm{HI}$ reaches 20 and a fast evolution stage after the $\mathrm{HI}$ exceeds 20 . The fault evolution within each stage is relatively stable, making the trajectory linear-like. As a result, the RS-GBDT model which was trained on these instances will capture these "stable" features and make flat RUL predictions during a certain period of time. However, the overall fault evolution is difficult to characterize and model due to its nonlinear, stochastic nature, hence a linear model may not be powerful enough to fully capture the characteristics of the degradation data. 2) The results produced by GBDT demonstrate stronger nonlinear data-RUL relationships than those of JITL-RS-GBDT, but at the cost of lower predictive accuracy. This indicates 
Table 2

Comparison of Prediction Interval Coverage Probability (PICP).

\begin{tabular}{|c|c|c|c|c|c|c|c|c|c|c|c|c|c|}
\hline Failure case no. & 1 & 2 & 3 & 4 & 5 & 6 & 7 & 8 & 9 & 10 & 11 & 12 & 13 \\
\hline JITL-RS-GBDT & $12 \%$ & $100 \%$ & $73 \%$ & $96 \%$ & $61 \%$ & $100 \%$ & $100 \%$ & $100 \%$ & $41 \%$ & $84 \%$ & $82 \%$ & $100 \%$ & $75 \%$ \\
\hline JITL-GBDT & $26 \%$ & $100 \%$ & $68 \%$ & $96 \%$ & $79 \%$ & $100 \%$ & $100 \%$ & $100 \%$ & $44 \%$ & $56 \%$ & $91 \%$ & $100 \%$ & $91 \%$ \\
\hline
\end{tabular}

Table 3

Comparison of the impact of distance measures on predictive accuracy.

\begin{tabular}{|c|c|c|c|c|c|c|c|c|c|}
\hline \multirow[t]{2}{*}{ Failure Cases } & \multicolumn{3}{|l|}{ MAE } & \multicolumn{3}{|l|}{ RMSE } & \multicolumn{3}{|l|}{ MAPE } \\
\hline & Proposed & DTW & Euclidean & Proposed & DTW & Euclidean & Proposed & DTW & Euclidean \\
\hline Case 1 & 63.7 & 122 & 122.89 & 71.8 & 145.6 & 145.9 & 0.395 & 0.71 & 0.708 \\
\hline Case 2 & 7.6 & 42.35 & 42.2 & 8.9 & 49.2 & 49.16 & 0.222 & 0.76 & 0.75 \\
\hline Case 3 & 40.7 & 25.53 & 27.1 & 57 & 49.31 & 50.53 & 0.256 & 0.17 & 0.185 \\
\hline Case 4 & 11.9 & 61.74 & 58.29 & 16.1 & 76.5 & 72.33 & 0.179 & 0.54 & 0.53 \\
\hline Case 5 & 15.9 & 21.75 & 21.67 & 17 & 34.8 & 34.21 & 0.531 & 0.328 & 0.307 \\
\hline Case 6 & 22.6 & 23.46 & 22.26 & 28.2 & 47.11 & 43.24 & 0.19 & 0.22 & 0.398 \\
\hline Case 7 & 9.9 & 18.27 & 18.66 & 12.2 & 29.47 & 29.73 & 0.409 & 0.62 & 0.62 \\
\hline Case 8 & 15.8 & 63.39 & 62.55 & 18.6 & 83.74 & 83.58 & 0.19 & 0.483 & 0.47 \\
\hline Case 9 & 110 & 92.88 & 108.03 & 149.7 & 131.4 & 148.9 & 0.414 & 0.44 & 0.509 \\
\hline Case 10 & 65.3 & 56.09 & 53.09 & 73.7 & 67.42 & 63.49 & 1.13 & 0.857 & 0.84 \\
\hline Case 11 & 8.1 & 60.95 & 63.23 & 11.7 & 79.36 & 80.45 & 0.178 & 0.491 & 0.529 \\
\hline Case 12 & 5.9 & 19.39 & 19.39 & 7 & 25.07 & 25.08 & 0.402 & 0.48 & 0.48 \\
\hline Case 13 & 33.8 & 75.51 & 80.04 & 42.1 & 101.4 & 103.11 & 0.60 & 0.494 & 0.585 \\
\hline
\end{tabular}

* Best results are highlighted in bold.

that the JITL module helps reduce the number of training instances, thereby allowing the RS-GBDT model to better capture the fault behaviour, preventing the unnecessary features from being learnt by the RS-GBDT model.

It is also worth noting that, in most cases, the prediction intervals in JITL-RS-GBDT appear to "converge" faster to the predicted RUL than those obtained by the JITL-GBDT model, which is a highly desirable attribute of a prognostic method. The reason could be attributed to the fact that the randomized and smoothed gradient have resulted in a predictive model that produces more tree splits when the prediction error is relatively large.

A metric called prediction interval coverage probability (PICP), defined by the percentage of true RUL that falls within the given RUL prediction interval, was computed to check the accuracy of confidence interval. The calculated PICP values were displayed in Table 2. The proposed method achieves similar PICP level as that of the JITL-GBDT model, demonstrating its capability to provide accurate confidence intervals.

In order to demonstrate the superiorities of the proposed similarity as stated in Eqs. (1)-(2), its effectiveness was verified through comparison with conventional distance measures, namely, Euclidean distance and dynamic time warping (DTW). The results were present in Table 3. It can be observed from the table that the proposed similarity metric could lead to better similarity matching thereby improving RUL prediction accuracy.

\section{Conclusions}

We developed a novel JITL-data-driven prognostic model to address the challenging problem of predicting RUL of compressors with diversified degradation patterns and various operational modes. Temperature measurements from the Head and Crank End discharge valves concerning thirteen failures were present. We detailed the theoretical advancement and practical implementation of the developed model. The proposed approach was compared with an existing GBDT model and an enhanced JITL-GBDT method. By leveraging the benefits of the JITL scheme, both the JITL-RS-GBDT and JITL-GBDT approaches outperformed the GBDT model in terms of predictive performance. Furthermore, the developed RS-GBDT model has shown superior performance over the GBDT algorithm in terms of both predictive accuracy and confidence intervals. Collectively, this study highlights the promise of JITL-data-driven models for RUL prediction of complex machines such as compressors.

In our previous publication [1] GBDTs are for the first time utilized for machinery prognostics. In this paper we proposed a JITL-RS-GBDT model which improves the traditional GBDTs in the following aspects. 1) the JITL-GBDT framework was for the first time proposed for machinery prognostics. This framework has proven to be better than GBDTs in terms of predictive accuracy due to the instance selection procedure employed. 2) The proposed improved JITL module selects source instances that fit better with the testing instance by filtering out these unrelated samples iteratively. The advantages in our JITL module is its capability of automatically determine the number of related instances without knowing the number of clusters centers/the number of instances need to be chosen, which are often required in traditional k-means-based instance selection method. 3) On the other hand, we propose a RS-GBDT model by putting forward new gradient and hessian functions, thereby 
allowing for more accurate RUL predictions and narrower confidence boundaries. This model offers the most attractive combinations of short training time, high accuracy and probabilistic outputs. The reason why GBDTs were used as base learners was that they are ensemble models with appealing characteristics, e.g. the ability to handle nonlinearity and not requiring the tedious feature pre-processing. It is a popular option for the machine learning tasks and particularly suitable for tasks where the feature number and sample size are relatively small (many real-world machine prognostics tasks are short of large number of historical failure cases).

The proposed prognostic model involves multi-sensor information fusion by principal component analysis. Only temperature measurements were utilized for the indication of fault evolution. In the future, other condition monitoring techniques, for instance vibration frequency domain analysis will be employed that provide with even earlier than temperature indications of fault extending the prognostic horizon.

\section{CRediT authorship contribution statement}

Xiaochuan Li: Writing - original draft, Conceptualization, Methodology, Software. David Mba: Supervision, Writing review \& editing. Tianran Lin: Supervision, Writing - review \& editing. Yingjie Yang: Supervision, Writing - review \& editing. Panagiotis Loukopoulos: .

\section{Declaration of Competing Interest}

The authors declare that they have no known competing financial interests or personal relationships that could have appeared to influence the work reported in this paper.

\section{References}

[1] X. Li, F. Duan, D. Mba, I. Bennett, Multidimensional prognostics for rotating machinery: A review, Adv. Mech. Eng. 9 (2) (2017) 1-20.

[2] Y. Lei, N. Li, L. Guo, N. Li, T. Yan, J. Lin, Machinery health prognostics: A systematic review from data acquisition to RUL prediction, Mech. Syst. Signal Process. 104 (2018) 799-834.

[3] M.S. Kan, A.C.C. Tan, J. Mathew, A review on prognostic techniques for non-stationary and non-linear rotating systems, Mech. Syst. Signal Process. 62 (2015) $1-20$.

[4] V.T. Tran, F. Althobiani, A. Ball, An approach to fault diagnosis of reciprocating compressor valves using Teager - Kaiser energy operator and deep belief networks, Expert Syst. Appl. 41 (9) (2014) 4113-4122.

[5] H.P. Bloch, A Practical Guide to Compressor Technology, Second. John Wiley \& Sons Inc, Hoboken, New Jersey, 2006.

[6] S. Yin, H. Gao, J. Qiu, O. Kaynak, Fault Detection for Nonlinear Process with Deterministic Disturbances: A Just-In-Time Learning Based Data Driven Method, IEEE Trans. Cybern. 47 (11) (2017) 3649-3657.

[7] C. Cheng, M. Chiu, Nonlinear process monitoring using JITL-PCA, Chemom. Intell. Lab. Syst. 76 (2005) 1-13.

[8] L. Yingchao, H. Xiaofeng, W. Zhang, Remaining useful life prediction based on health index similarity, Reliab. Eng. Syst. Saf. 185 (2019) 502-510.

[9] T. Wang, J. Yu, D. Siegel, and J. Lee, "A similarity-based prognostics approach for Remaining Useful Life estimation of engineered systems," in Prognostics and Health Management, 2008. PHM 2008. International Conference on, 2008, pp. 1-6.

[10] E. Zio, F. Di Maio, A data-driven fuzzy approach for predicting the remaining useful life in dynamic failure scenarios of a nuclear system, Reliab. Eng. Syst. Saf. 95 (1) (2010) 49-57.

[11] P. Loukopoulos et al, Abrupt fault remaining useful life estimation using measurements from a reciprocating compressor valve failure, Mech. Syst. Signal Process. 121 (2019) 359-372.

[12] T. Loutas N. Eleftheroglou G. Georgoulas P. Loukopoulos D. Mba I. Bennett Valve Failure Prognostics In Reciprocating Compressors Utilizing Temperature Measurements, PCA-based Data Fusion And Probabilistic Algorithms IEEE Trans. Ind. Electron. 201911

[13] T. Wang, "Trajectory Similarity Based Prediction for Remaining, Useful Life Estimation" (2010).

[14] L. Wu, "Random Warping Series: A Random Features Method for Time-Series Embedding," vol. 84, 2018.

[15] Q. Lei, J. Yi, R. Vaculin, L. Wu, I.S. Dhillon, “Similarity preserving representation learning for time series clustering”, IJCAI Int. Jt. Conf, Artif. Intell. vol. 2019-Augus (2019) 2845-2851.

[16] K.P. Murphy, Machine Learning - A Probabilistic Perspective -, Table-of-Contents. (2012).

[17] L. Liao, F. Köttig, A hybrid framework combining data-driven and model-based methods for system remaining useful life prediction, Appl. Soft Comput. J. 44 (2016) 191-199.

[18] M. Ester, H. Kriegel, X. Xu, D-. Miinchen, A Density-Based Algorithm for Discovering Clusters in Large Spatial Databases with Noise, AAAI conference (1996).

[19] J. Wu, C. Wu, S. Cao, S.W. Or, C. Deng, X. Shao, Degradation Data-Driven Time-To-Failure Prognostics Approach for Rolling Element Bearings in Electrical Machines, IEEE Trans. Ind. Electron. 66 (1) (2018) 529-539.

[20] P. Loukopoulos, P. Pilidis, I. Bennett, G. Zolkiewski, L. Xiaochuan, D. Mba, Abrupt fault remaining useful life estimation using measurements from a reciprocating compressor valve failure, Mech. Syst. Signal Process. (2018).

[21] L. Breiman, Bagging Predictors, Mach. Learn. 24 (421) (1996) 123-140.

[22] R.E. Schapire, The Strength of Weak Learnability, Mach. Learn. 5 (2) (1990) 197-227.

[23] L. Breiman, Random forests, Mach. Learn. 45 (2001) 5-32.

[24] Y. Freund, R.E. Schapire, A Decision-Theoretic Generalization of On-Line Learning and an Application to Boosting, J. Comput. Syst. Sci. 55 (1997) 119 139.

[25] L. Breiman, Prediction games and arcing algorithms, Neural Comput. 11 (7) (1998) 1-30.

[26] Jerome H. Friedman, Greedy function approximation: a gradient boosting machine, Ann. Stat. (2001) 1189-1232.

[27] N. Meinshausen, Quantile Regression Forests, J. Mach. Learn. Res. 7 (2006) 983-999.

[28] A. Saxena, K. Goebel, D. Simon, and N. Eklund, "Damage propagation modeling for aircraft engine run-to-failure simulation," in 2008 International Conference on Prognostics and Health Management, PHM 2008, 2008.

[29] T. Chen and C. Guestrin, "XGBoost: A scalable tree boosting system," in Proceedings of the ACM SIGKDD International Conference on Knowledge Discovery and Data Mining, 2016, vol. 13-17-Augu, pp. 785-794.

[30] S. Hochreiter, J. Schmidhuber, Long short-term memory, Neural Comput. 9 (8) (1997) 1735-1780. 